\title{
ANÁLISIS ECONÓMICO DE LA DIFERENCIA EN LA PROBABILIDAD DE TRABAJO INFANTIL ENTRE AFROCOLOMBIANOS Y NO AFROCOLOMBIANOS
}

\author{
Ernesto Cardenas Prieto * \\ Bladimir Carabalí Hinestroza ${ }^{\dagger}$
}

\begin{abstract}
Resumo
Usamos la técnica de descomposición de Yun (2003) con el fin de analizar la diferencia de probabilidad de trabajo infantil entre Afrocolombianos y No Afrocolombianos. Encontramos que dicha diferencia es de 3,2\% y que el 91,6\% de la misma es explicada por el efecto características mientras que el restante $8,4 \%$ lo explica el efecto coeficientes. La característica socioeconómica más importante para explicar dicha diferencia es la condición urbano-rural que explica un $46 \%$. Con respecto al efecto coeficientes proponemos un modelo que incorpora discriminación laboral como una posible explicación de la mayor tasa de trabajo infantil de los niños Afrocolombianos.
\end{abstract}

Palabras clave: Trabajo infantil, discriminación, desarrollo económico.

\begin{abstract}
We use Yun (2003)'s decomposition in order to analyze the difference in probability terms of child labor for two ethnic groups Afro-Colombians and Non Afro-Colombians. We found this difference to be of $3,2 \%$. Also, we found that $91,6 \%$ of the difference is explained by the so called characteristics effect and $8,4 \%$ by the coefficients effect.

The most important socioeconomic characteristic at explaining the difference is the urban-rural condition, which explains $46 \%$. Regarding the coefficients effect, we propose a model of labor discrimination as a possible explanation for the higher probability of child labor into the AfroColombian group.
\end{abstract}

Keywords: Child labor, discrimination, development economics.

JEL classification: D13, J71, O12.

DOI: http://dx.doi .org/10.11606/1413-8050/ea139913

\footnotetext{
* Profesor Asociado. Departamento de Economía. Pontificia Universidad Javeriana Cali. Colombia. E-mail: ernesto.cardenas@javerianacali.edu.co

${ }^{\dagger}$ UNICAMP - Universidad Estadual de Campinas. Programa de Post-Graduación en Demografía. Campinas, San Paulo, Brasil. E-mail: bladimir.carabali@nepo.unicamp.br
} 


\section{Introducción}

El problema del trabajo infantil a nivel mundial es sin duda un tema preocupante. De acuerdo con el informe de la Ofcina Internacional del Trabajo (2013), casi un $11 \%$ del total de la población infantil se encontraba trabajando en el año 2012, es decir, 168 millones de niños entre los 5 y los 17 años de edad. En América Latina y el Caribe, según el mismo informe, el $9 \%$ de la población infantil trabaja, es decir, 12.5 millones de niños.

$\mathrm{Al}$ analizar esta problemática, la mayoría de los trabajos en economía investigan la participación de diferentes características socioeconómicas sobre las tasas de trabajo infantil y encuentran una relación estrecha entre pobreza y trabajo infantil. Hogares más pobres están asociados con mayores niveles de trabajo infantil (Basu \& Van 1998, Ray 2000b).

Sin embargo, la posible relación entre trabajo infantil y afrodesendencia no ha sido explorada ni en la literatura internacional ni en la colombiana, a pesar de que existe evidencia de diferencias en términos de ingresos entre estos grupos poblacionales (Gradín 2007, 2010). En otras palabras, el siguiente tipo de preguntas no ha sido abordado: ¿existe alguna diferencia en la tasa de trabajo infantil entre afrodescendientes y no afrodescendientes? Si existe alguna diferencia ¿qué características socioeconómicas explican dicha diferencia?

El objetivo de este documento es precisamente el de estudiar la diferencia en la probabilidad de trabajo infantil entre dos grupos poblacionales: Afrocolombianos y No Afrocolombianos. En concreto, se realiza una descomposición de la diferencia de la tasa de trabajo infantil entre los dos grupos propuestos para saber si dicha diferencia obedece principalmente a las características socioeconómicas de los grupos o a la estructura económica y social Colombiana. La técnica de descomposición que se utiliza es la técnica de descomposición de Yun (2003), ya que permite calcular los denominados efectos características y coeficientes, asociados precisamente con las contribuciones de las características de los grupos y con factores culturales o estructurales respectivamente. Adicionalmente esta técnica puede ser aplicada cuando la variable dependiente es dicotómica como lo es en nuestro caso.

El trabajo continúa en la sección 2 con una revisión bibliográfica en torno al tema del trabajo infantil. En la sección 3 se presenta en detalle la metodología de descomposición de Yun, mientras que en la sección 4 se describen los datos utilizados en el trabajo. Los resultados del trabajo se analizan en la sección 5 y las conclusiones del trabajo se presentan en la sección 6 .

\section{Revisión Bibliográfica}

Siguiendo a Myers (2001) se puede decir que el tema del trabajo infantil ha sido abordado desde cuatro grandes perspectivas. La primera, aborda la problemática del trabajo infantil desde el funcionamiento del mercado laboral; la segunda, desde la perspectiva del capital humano como factor de desarrollo económico; la tercera, desde la perspectiva de la responsabilidad social y, la cuarta, desde el punto de vista de la niñez.

Desde el funcionamiento del mercado laboral, se estudia la posibilidad de que el trabajo infantil desplace al trabajo adulto. Para Marx (1999), características de los niños como el tamaño de su cuerpo, los hacen apropiados para ciertos trabajos específicos, por ejemplo, en el sector agrícola. En ese 
mismo sentido Levy (1985) encuentra en Egipto una relación positiva entre el incremento del número de niños en el hogar y la producción agrícola. Recientemente trabajos como los de Swaminathan (1988), Admassie (2003) que abordan el mismo problema encuentran relaciones similares en Etiopía e India, respectivamente.

Desde la segunda perspectiva, la del capital humano, se considera al trabajo infantil como un producto del subdesarrollo, que tiende a desaparecer a medida que los países reducen sus condiciones de pobreza y desigualdad. Ray (2000a), Wahba (2000) analizan la relación entre pobreza y trabajo infantil y encuentran que la pobreza estimula la participación laboral de los niños y reduce la posibilidad de que estudien.

Según Ndjanyou \& Djiénouassi (2010), en Camerún, condiciones socioeconómicas, demográficas y de localización geográfica del hogar juegan un papel importante en la determinación del trabajo infantil. En términos del género del jefe del hogar, cuando los hogares presentan una mujer como jefe de hogar se incrementa el riesgo de trabajo infantil. Por el lado socioeconómico la informalidad laboral así como los bajos niveles educativos del jefe de hogar hacen más probable que un niño ingrese al mercado laboral.

Tharmmapornphilas (2008) muestra, para Thailandia, que el ingreso del jefe de hogar es el determinante más importante del trabajo infantil. Mayores niveles de ingresos del jefe de hogar disminuyen el riesgo de trabajo infantil. Además, señala la importancia de la localización geográfica, ya que estar en la zona rural (caracterizada por mayores niveles de pobreza) aumenta el riesgo del trabajo infantil.

Zapata et al. (2011) también encuentran, para Bolivia, que salarios y niveles educativos bajos del jefe de hogar aumentan la probabilidad de trabajo infantil. Adicionalmente, que los varones tienen una mayor probabilidad de ingresar al mercado laboral, mientras que las mujeres doblan la probabilidad de trabajar en el hogar con respecto a la de los hombres. Finalmente, señalan que los niños indígenas tienen menor probabilidad de asistir a la escuela con respecto a otros grupos poblacionales de Bolivia. Ray (2001), Ersado (2002) muestran que las características socioeconómicas de los hogares afectan de manera simultánea la decisión entre asistencia escolar y trabajo infantil al interior de los hogares. Un resultado importante en estos trabajos es que un mayor nivel educativo del jefe de hogar disminuye el trabajo infantil e incrementa la educación del niño.

Desde el punto de vista de la responsabilidad social, el trabajo infantil es considerado como el resultado de una combinación de factores, tales como la discriminación cultural, la desigualdad de oportunidades y la concentración de poder. Emerson \& Knabb (2006), por ejemplo, encuentran evidencia empírica de que la persistencia intergeneracional del trabajo infantil no se debe atribuir sólo a la pobreza económica sino a las diferencias en términos de oportunidades, en la calidad de la educación, de acceso de los jefes de hogar a trabajos con salarios altos, de acceso a la información y de discriminación. Finalmente, cuando la problemática se analiza, desde los intereses propios de la niñez, los derechos de los niños, su desarrollo y bienestar son los ejes fundamentales del análisis.

O'Donnel et al. (2002), para Vietnam, encuentran que el trabajo infantil en el sector agrícola tiene efectos negativos en la salud de los niños a largo plazo. En ese mismo sentido, Kassouf et al. (2001) señalan que ingresar a trabajar 
en Brasil a temprana edad aumenta la probabilidad de reportar problemas de salud en la etapa de la adultez.

\subsection{Estudios Realizados en Colombia}

En Colombia, trabajos como los de Bernal \& Cárdenas (2006), Pedraza \& Ribero (2005) señalan que los factores que más influyen sobre la probabilidad del trabajo infantil son los asociados con la pobreza, el nivel educativo de los padres, las condiciones demográficas del niño (género y edad principalmente) y la localización geográfica (zona rural).

El estudio de Pedraza \& Ribero (2006) concluye que el trabajo infantil tiene un efecto negativo sobre la salud y la educación de los niños. Tovar et al. (2009), para el departamento del Valle del Cauca, muestran que existe un trade-of entre trabajo infantil y asistencia escolar, es decir, los niños que trabajan disminuyen su probabilidad de asistir a la escuela.

Por último, el trabajo de Ávila (2009), observa que efectivamente el trabajo infantil responde a un razonamiento distinto si el trabajo es realizado fuera o dentro del hogar. En el caso de trabajo fuera del hogar, se cumple la hipótesis de que a menor riqueza mayor trabajo infantil; mientras en el caso del trabajo en un negocio familiar, la relación es la opuesta, a mayor riqueza mayor trabajo infantil, puesto que en este caso el trabajo infantil es visto como etapa de formación para los niños.

Es importante destacar que, en general, los estudios sobre trabajo infantil se preocupan por el efecto de las variables demográficas, geográficas y económicas sobre el propio trabajo infantil. Sin embargo, la diferencia del efecto de estas características sobre distintos grupos sociales no ha recibido suficiente atención, y por lo tanto el presente trabajo es un aporte valioso en ese sentido.

En general, todos los estudios anteriores señalan la importancia o el efecto de las variables demográficas, geográficas y económicas sobre el trabajo infantil. Sin embargo, la diferencia del efecto de estas características en diferentes grupos sociales tampoco ha sido estudiada en Colombia.

\section{Metodología}

Diferentes técnicas de descomposición han sido propuestas a partir del trabajo seminal de Oaxaca (1973), Blinder $(1973)^{1}$. En este trabajo utilizamos la técnica descomposición no lineal propuesta por Yun (2003) en atención a la característica dicotómica de nuestra variable dependiente y, además, a que esta técnica nos permite descomponer la diferencia en la probabilidad de trabajo infantil entre Afrocolombianos y no Afrocolombianos en dos efectos, denominados características y coeficientes, que están asociados a las características socioeconómicas de cada uno de los grupos y a la estructura social o cultural relevante (objetivo del presente trabajo). Técnicas de descomposición no lineales como la de Fairlie (2005) se enfocan solamente en la denominada descomposición detallada o de características y no permiten la descomposición entre efecto características y coeficientes.

La técnica de descomposición de Yun está basada en la técnica de descomposición de Blinder y Oaxaca. La idea desarrollada por estos últimos es que

\footnotetext{
${ }^{1}$ Un análisis detallado de los diferentes métodos de descomposición en economía se encuentra en: Fortin et al. (2011).
} 
la diferencia en términos de la variable dependiente entre diferentes grupos puede ser explicada por la diferencia en sus características y por la diferencia en los coeficientes estimados a través de mínimos cuadrados ordinarios para cada una de las características en cada uno de los grupos.

El efecto características tiene que ver con la posibilidad de hacer análisis contrafactual. De hecho, el efecto características corresponde a la medición del desempeño que tendría uno de los grupos objeto de estudio (desempeño en la variable dependiente), si se le otorgaran las características (variables independientes) del otro grupo. Así, el efecto características resulta importante, por ejemplo, a la hora de tomar decisiones de política económica, ya que permite identificar características puntuales sobre las que se deberían enfocar dichas políticas.

Por otra parte, el efecto coeficientes o efecto no explicado está asociado con elementos estructurales de una sociedad, tales como factores culturales o estructuras de poder entre otros, los cuales hacen que diferentes grupos sociales obtengan diferentes resultados. El análisis del efecto coeficientes está asociado entonces con políticas de largo plazo que logren afectar la estructura misma de la sociedad.

Esta descomposición en efecto características y en efecto coeficientes resulta inmediata cuando la forma funcional que relaciona las variables es de tipo lineal. En caso contrario, para funciones no lineales, surge el siguiente interrogante: ¿cuáles son los pesos por los cuales se debe ponderar la contribución de cada característica al efecto características y al efecto coeficientes? Yun (2003) propone una descomposición para el caso de funciones no lineales, basada en la descomposición Oaxaca- Blinder, en la que primero evalúa el valor de la función en los valores promedio de las características y, segundo, a través de una expansión de Taylor de primer orden linealiza los efectos características y coeficientes alrededor de la media de cada uno de los grupos.

Con esta técnica examinamos la contribución de diferentes características socioeconómicas de los hogares a la diferencia en la probabilidad de trabajo infantil entre Afrocolombianos y no Afrocolombianos. Denotamos con $y_{i}$ la variable dependiente del i-ésimo individuo, trabajo infantil, y asumimos que solo puede tomar dos valores así: $y_{i}=1$ si el $i$-ésimo niño trabaja y $y_{i}=0$ si el $i$-ésimo niño no trabaja. El grupo racial al cual pertenece el niño será denotado con $j=0,1$. Donde $j=0$ denota el grupo de no Afrocolombianos $j=1$ el de Afrocolombianos.

Así, bajo un modelo probabilístico no lineal tipo Logit la probabilidad de que un niño en el grupo $j$ se encuentre trabajando está dada por:

$$
p_{j}^{i}=\operatorname{prob}\left(y_{i}^{j}=1\right)=F\left(X_{i}^{j} \tilde{\beta}^{j}\right)=\frac{\exp \left(x_{i}^{j} \tilde{\beta}^{j}\right)}{1+\exp \left(x_{i}^{j} \tilde{\beta}^{j}\right)}
$$

donde $F$ simboliza la distribución de probabilidad acumulativa de una función logística, $X_{i}^{j}$ es un vector de características del hogar del niño $i$ y tilde $\beta$ es el vector asociado de coeficientes estimados.

En el marco del modelo Logit, la proporción de trabajo infantil en el grupo $j$, es decir $y^{j}$, es igual a la probabilidad predicha para ese grupo con población $N^{j}$.

Por lo tanto, 


$$
Y^{j}=\overline{P^{j}}=\overline{F\left(X_{i}^{j} \tilde{\beta}^{j}\right)}=\frac{1}{N^{j}} \sum_{i=1}^{N^{j}} F\left(X_{i}^{j} \beta^{j}\right)
$$

Este tipo de relaciones no lineales se pueden descomponer así:

$$
Y_{1}-Y_{0}=\left[\overline{F\left(X_{1} \beta_{1}\right)}-\overline{F\left(X_{0} \beta_{1}\right)}+\overline{F\left(X_{0} \beta_{1}\right)}-\overline{F\left(X_{0} \beta_{0}\right)}\right]
$$

La descomposición en la ecuación (3) es la suma del efecto características $\overline{F\left(X_{1} \beta_{1}\right)}-\overline{F\left(X_{0} \beta_{1}\right)}$ y del efecto coeficientes o diferencia no explicada $\overline{F\left(X_{0} \beta_{1}\right)}-$ $\overline{F\left(X_{0} \beta_{0}\right)}$. El efecto características es la diferencia explicada por las características de cada grupo, mientras que el efecto coeficientes refleja la diferencia debida al rendimiento diferente que pueden tener las mismas características en cada uno de los diferentes grupos.

A partir de esta descomposición conjunta Yun establece la contribución individual de cada variable a la diferencia de la siguiente manera:

$$
Y_{1}-Y_{0}=\sum_{K=1}^{K} W_{\nabla X}^{K}\left[\overline{F\left(X_{1} \beta_{1}\right)}-\overline{F\left(X_{0} \beta_{1}\right)}\right]+\sum_{K=1}^{K} W_{\nabla \beta}^{K}\left[\overline{F\left(X_{0} \beta_{1}\right)}-\overline{F\left(X_{0} \beta_{0}\right)}\right]
$$

Donde $W_{\nabla X}^{K}$ y $W_{\nabla \beta}^{K}$ son las contribuciones individuales relativas de la característica $\mathrm{k}$ al efecto características y al efecto coeficientes respectivamente así:

$$
W_{\nabla X}^{K}=\frac{\left(\bar{x}_{k}^{1}-\bar{x}_{k}^{0}\right) \beta_{k}^{1}}{\left(\bar{x}^{1}-\bar{x}^{0}\right) \beta^{1}} ; W_{\nabla \beta}^{K}=\frac{\left(\beta_{k}^{1}-\beta_{k}^{0}\right) \bar{x}_{k}^{0}}{\left(\beta^{1}-\beta^{0}\right) \bar{x}^{0}} ; \sum_{K=1}^{K} W_{\nabla X}^{K}=1 ; \sum_{K=1}^{K} W_{\nabla \beta}^{K}=1
$$

Estos pesos fueron obtenidos por Yun utilizando dos estrategias: (i) el valor medio de $F, \overline{F\left(X_{h} \beta_{h}\right)}$, se aproxima a la función evaluada en el promedio de la muestra de las variables, $F\left(\overline{X_{h} \beta_{h}}\right)$; y (ii) se utiliza la expansión de Taylor de primer orden para linealizar los efectos asociados las características y a los coeficientes entorno a la media.

Por último, es importante mencionar el problema de identificación presente en la descomposición detallada del efecto coeficientes; el cual se presenta con variables categóricas o dummies, porque las contribuciones de estas variables cambian cuando la variable cambia de grupo de referencia. Este problema requiere el cálculo previo de una regresión normalizada que corrija el problema de identificación (Yun 2005).

\section{Datos}

Los datos utilizados provienen de la Encuesta de Calidad de Vida (ECV) del 2003, realizada por el Departamento Administrativo Nacional de Estadística de Colombia (DANE) ya que son los únicos datos para los cuales se tiene en forma simultánea información sobre trabajo infantil y la condición racial de las personas. A continuación se describen los datos utilizados y se presentan las principales estadísticas descriptivas obtenidas. 
La encuesta es de cobertura nacional y cuenta con un registro de 22.949 hogares seleccionados mediante diseños probabilísticos. Para este trabajo se utilizaron solamente 21.744 hogares de la muestra $(94,74 \%)$ que corresponden a hogares con niños entre 5-17 años.

Del total de niños un $12,9 \%$ se encontraban realizando un trabajo. Del total de niños en la muestra $50,3 \%$ son hombres y $49,5 \%$ mujeres. Del total de hombres un $11,9 \%$ trabajaban, mientras que de las mujeres lo hacían el 12,1 La situación del trabajo infantil por regiones (departamentos) se resume en la Tabla 1 en los anexos. Se destaca que la región Pacífica concentra el porcentaje más alto de trabajo infantil, 21,0\%, seguida de las regiones Central y Oriental con 15,5\%. Por otra parte, se observa que San Andrés y Providencia, junto con Bogotá, presentan las tasas más bajas de trabajo infantil, 4,8\% y $7,3 \%$ respectivamente.

Con respecto a los jefes de hogar encontramos que un $28,9 \%$ son mujeres y un $71,1 \%$ son hombres con ingresos promedio mensuales de $\$ 235.500 \mathrm{y}$ $\$ 252.593$ pesos colombianos, respectivamente (US\$ 82 en el primer caso y US\$ 88 dólares en el segundo, a precios constantes de 2003).

Un análisis por condición étnico-racial permite ver que los hogares Afrocolombianos presentan un mayor porcentaje de pobreza, 70,3\%, comparado con un $64,2 \%$ de los no Afrocolombianos, y que la región Pacífica, que es la de mayor concentración de población Afrocolombiana, registra la proporción más alta de hogares pobres, 79,6\% (ver Tabla A.2. En relación a los niveles educativos se observa que el $17,3 \%$ de los jefes de hogares Afrocolombianos no tienen ningún nivel educativo, mientras que en los no Afrocolombianos el mismo porcentaje es tan sólo de 10,4\%.

En términos del mercado laboral, la diferencia entre los jefes de hogares Afrocolombianos que tienen trabajo formal y los no Afrocolombianos es de solamente el $1 \%$ en contra de los primeros. Finalmente, es importante señalar que el $37,9 \%$ de los hogares Afrocolombianos se encontraban en la zona rural mientras sólo un $28,7 \%$ de los no Afrocolombianos lo hacían. La descripción de las variables usadas en el trabajo se presenta en la Tabla A.3.

\section{Resultados}

\subsection{Efecto Características}

La diferencia entre la probabilidad de que un niño Afrocolombiano se encuentre trabajando, con respecto a un niño no Afrocolombiano, es de 3,2\% (Tabla A.4).

El resultado de la descomposición de Yun en efecto características y efecto coeficientes de la diferencia en la tasa de trabajo infantil entre los dos grupos aparece reportado en la Tabla 5 en los anexos del trabajo. Mirando la tabla se observa que el efecto características explica el 91,6\% de la diferencia mientras que el efecto coeficientes explica el 8,4\% restante. Este resultado se puede interpretar de manera contrafactual así: si le otorgáramos al grupo de los niños Afrocolombianos las características del grupo de niños no Afrocolombianos, la diferencia en la tasa de trabajo infantil pasaría de 3,2\% a 0,29\% entre los dos grupos.

La descomposición detallada del efecto características, que también se reporta en la Tabla A.5, señala la importancia principal de tres características 
para explicar el total de la diferencia: la condición urbano-rural, el nivel educativo del jefe de hogar y el tamaño del hogar.

La condición urbano-rural explica el $46 \%$ de la diferencia. Este resultado evidencia la enorme disparidad entre el mundo rural y el urbano en Colombia. Por ejemplo, en las zonas rurales la falta de oportunidades, no solo de educación, sino en general de actividades recreativas y culturales aumentan los incentivos para que los niños tengan que ir a trabajar. Adicionalmente, en las regiones rurales el trabajo infantil puede ser asimilado como algo culturalmente aceptable a diferencia de las zonas urbanas.

La importancia de la característica urbano-rural implica, en términos de política económica que políticas encaminadas a mejorar las condiciones y expectativas de vida en las zonas rurales deberían tener un impacto significativo en la reducción de tasas de trabajo infantil, en general, y en la reducción de la brecha de trabajo infantil entre Afrocolombianos y no Afrocolombianos, en particular.

La segunda característica socioeconómica en importancia es el nivel educativo del jefe de hogar. Esta característica explica el 29,6\% de la diferencia. Jefes de hogar con mayor nivel educativo posiblemente prefieren que sus hijos ingresen al sistema educativo y no al mercado laboral.

Por otra parte el tamaño de los hogares explica casi un 10\% de la diferencia. Hogares más grandes enfrentan mayores requerimientos económicos para su supervivencia, lo cual los hace eventualmente más vulnerables al trabajo infantil.

Además de la importancia en términos económicos de las tres variables socioeconómicas apenas descritas también puede observarse, en la misma Tabla A.2, que estas variables son estadísticamente significativas.

Las Tablas A.6 y A.7, reportan la descomposición también en efecto características y efecto coeficientes para cada uno de los dos grupos, Afrocolombianos y no Afrocolombianos respectivamente. Los resultados indican la importancia de las mismas 3 características.

\subsection{Efecto Coeficientes}

Como se mencionó anteriormente el efecto coeficientes explica un $8,4 \%$ de la diferencia en la tasa de trabajo infantil entre los dos grupos y está asociado con características estructurales de la sociedad. Es decir, existen estructuras al interior de la sociedad que pueden estar afectando, en este caso particular, la tasa de trabajo infantil. Dada la importancia práctica que esto tiene, a pesar de que el efecto no resulta estadísticamente significativo, ofrecemos una posible explicación teórica para la interpretación de este resultado. En concreto, nos ocupamos de una de las posibles estructuras sociales que pueden existir detrás del efecto coeficientes, a saber, la de una posible discriminación laboral en trabajos calificados en contra de la población afrocolombiana. Lo anterior, en atención a evidencia reportada en trabajos previos sobre la posible existencia de dicho fenómeno en Colombia.

De hecho, Tenjo \& Herrera (2009) señalan que los Afrocolombianos presentan dificultades de acceso a las ocupaciones mejor pagadas, lo cual podría explicarse por factores de discriminación en el mercado laboral. Portilla (2003) señala que los Afro descendientes situados en la ciudad de Cali, tienen menores probabilidades de encontrarse en empleos formales, pero consideran que dicho efecto obedece a que presenta menor capital humano que la población 
blanca-mestiza, es decir la discriminación se podría presentar en el acceso a la educación superior. Por último, el trabajo de Viáfara \& Urrea (2006), señala que las diferencias en el logro educativo son significativas entre Afrocolombianos y no Afrocolombianos, en otras palabras, las personas Afrocolombianas tienen menores probabilidades de alcanzar un nivel educativo alto. Adicionalmente encuentran que los Afrocolombianos tienen menos probabilidades de alcanzar un alto estatus socio ocupacional.

Por discriminación laboral entendemos aquella que ocurre cuando algunos grupos sociales reciben trato diferencial, dependiendo de alguna característica, e independiente de sus capacidades para desempeñar sus actividades laborales. Este trato diferencial puede verse reflejado, por ejemplo, en diferencias salariales: el grupo discriminado recibe un menor sueldo a pesar de exhibir las mismas condiciones de capital humano y posición ocupacional ${ }^{2}$. En economía, dos teorías basadas en los fallos de mercados competitivos plantean explicaciones al fenómeno.

\section{Modelo para Explicar el Efecto Coeficientes}

A continuación se presenta un modelo teórico sencillo, con el ánimo de proporcionar una estructura que permita interpretar los resultados obtenidos asociados al efecto coeficientes. Es importante aclarar que no se pretende obtener del modelo una ecuación que posteriormente sea estimable a través de métodos econométricos, sino que el modelo provee un marco para la interpretación de los resultados obtenidos.

En este modelo el jefe de hogar toma las decisiones sobre la asignación del tiempo de sus hijos con el ánimo de maximizar el nivel de utilidad del hogar. En particular, suponemos que solamente existen 2 periodos de tiempo, presente y futuro, que el jefe de hogar debe decidir en el presente, si sus hijos van a estudiar o a trabajar ${ }^{3}$. Suponemos que independientemente de esta decisión, los hijos en el segundo periodo, necesariamente tienen que trabajar. La utilidad del hogar está representada en términos de consumo total y equivale a la suma de los consumos en cada uno de los períodos $C_{1}$ (consumo presente) y $C_{2}$ (consumo futuro).

\footnotetext{
${ }^{2}$ Para Becker (1971), no puede existir discriminación en mercados competitivos. En los mercados competitivos la interacción entre oferta y demanda garantiza que los trabajadores sean remunerados según su productividad marginal. Trabajadores más competitivos reciben un mayor salario con respecto a aquellos que no lo son. La productividad del trabajador es pues la única característica relevante para determinar los salarios y no implica discriminación alguna. Por lo tanto, la discriminación solo puede ocurrir en mercados no competitivos en los que es posible que exista un gusto por la discriminación.De otra parte, la teoría de la discriminación estadística planteada por Arrow \& Kenneth (1972) se basa en la existencia de problemas de información. En concreto, señala que la discriminación en el mercado laboral obedece a un problema de información en el momento de contratar a un trabajador. Es decir, que los empleadores no pueden identificar las variables necesarias que permiten dar cuenta de las potencialidades de los aspirantes al trabajo, por lo tanto, la señal del grupo social al cual pertenece un individuo se convierte en información suficiente para la realización de las valoraciones necesarias. Por otra parte, la discriminación se presenta en la medida que algunos grupos específicos de la sociedad no reciben las mismas dotaciones que el resto de individuos, por ejemplo, la calidad de la educación que es determinante para efecto de la movilidad social u obtención de buenos salarios.

${ }^{3}$ El modelo supone que el trabajo y el estudio son mutuamente excluyentes. A pesar de que los niños pueden trabajar y estudiar al tiempo, principalmente en las zonas rurales, el supuesto captura la idea de que una educación de calidad, que es la que permite posterior acceso al trabajo calificado, generalmente requiere dedicación exclusiva.
} 
Se supone que la utilidad del hogar aumenta con el nivel de consumo total. Los ingresos del hogar en cada periodo de tiempo pueden provenir de dos fuentes, la remuneración al trabajo realizado por el jefe del hogar y la remuneración al trabajo realizado por el hijo del hogar y que denotamos con $m_{j}^{t} \mathrm{y}$ $m_{h}^{t}$ respectivamente para $t=1,2$. Así, por ejemplo, si el jefe del hogar es el único que trabaja en el presente, $t=1$, entonces el total de ingreso disponible para consumo en el hogar será $m_{j}^{1}$ pero si el jefe del hogar decide que su hijo trabaje entonces el ingreso total del hogar será $m_{j}^{1}+m_{h}^{1}$.

Asumimos por simplicidad que cada hogar tiene solamente un hijo, aunque el modelo podría ser generalizado para el caso de varios hijos, y que los niveles de precios del consumo se mantienen constantes e iguales a 1.

Si el jefe del hogar decide que su hijo trabaje en el presente, aumentando el ingreso y las posibilidades de consumo presente del hogar, entonces incurre en un costo $\delta$. Este valor representa el costo de oportunidad que implica trabajar en el presente y no acceder a trabajos con mayor remuneración en el futuro, y que están destinados solamente a personas que decidieron estudiar en el primer periodo. La posible existencia de discriminación en el mercado laboral para personas calificadas basada en la condición étnico-racial de las persona, se modela a través de una función de $\operatorname{costos} \delta\left(\theta^{i}\right) \operatorname{con} i=n, b$. En particular, asumimos que es una función de la condición étnico-racial de las personas así: $\delta\left(\theta^{b}\right) \geq \delta\left(\theta^{n}\right)$, donde $\theta^{b}$ y $\theta^{n}$ indican si la condición étnico-racial del individuo es afrodescendiente o no, respectivamente. El cumplimiento estricto de la desigualdad anterior implica la existencia de discriminación laboral en contra de la población afrodescendiente, mientras que la igualdad implicaría que no hay discriminación.

Asumimos aquí, que los hogares no tienen acceso al mercado crediticio, y por lo tanto no pueden pedir dinero prestado, así que su consumo depende exclusivamente de los ingresos generados por su trabajo.

Dado lo anterior, podemos derivar la restricción presupuestaria que enfrenta el jefe del hogar de la siguiente manera: si el jefe del hogar decide que su hijo trabaje en $t=1$ entonces $C_{1}>m_{j}^{1}$, es decir, el nivel de consumo presente del hogar es mayor a la remuneración obtenida por el jefe del hogar en dicho periodo. De hecho, la diferencia entre consumo e ingresos será igual al salario devengado por el hijo, es decir, $m_{h}^{1}=\left(C_{1}-m_{j}^{1}\right.$. Dado que el hijo no va a poder acceder a trabajos calificados en el futuro, esta decisión implica un costo de oportunidad dado por $\delta\left(C_{1}-m_{j}^{1}\right)$. Por otra parte, si el jefe de hogar decide que su hijo estudie en $t=1$; entonces, sus posibilidades de consumo en $t=1$ serán $C_{1}<m_{j}^{1}$ asumiendo que parte del ingreso $m_{j}^{1}$ debe destinarse a cubrir los costos de educación. Esta decisión, sin embargo, implica un aumento en el consumo en el periodo $t=2$ de $\delta\left(m_{j}^{1}-C_{1}\right)$, ya que el hijo podría acceder (si no hay discriminación) a trabajos calificados con mayor remuneración. Podemos escribir entonces la restricción presupuestaria como:

$$
c_{2}=2 m_{j}^{2}+\delta \cdot\left(m_{j}^{1}-c_{1}\right)
$$

La restricción presupuestaria se representa en el gráfico 1. 


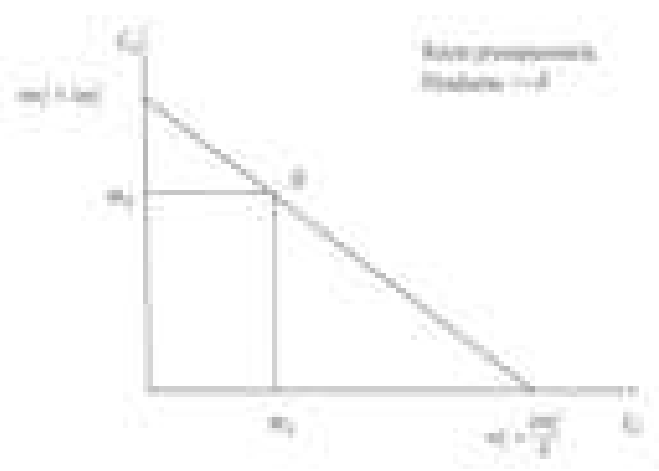

Figura 1: Restricción Presupuestaria

\section{Estática comparativa}

Asumimos que el jefe de hogar tiene unas preferencias por el consumo, que pueden ser representadas a través de una función de utilidad estrictamente cóncava, es decir, que está dispuesto a sustituir consumo presente por consumo futuro. Así, si el jefe de hogar elige un nivel de consumo tal que $C_{1}>m_{j}^{1}$, entonces, dado que el nivel de consumo óptimo excede el ingreso del jefe del hogar, decimos que existen incentivos para que el niño trabaje en el período 1. Si el jefe de hogar elige un nivel de consumo tal que $C_{1}<m_{j}^{1}$, entonces, dado que el nivel de ingreso del jefe del hogar excede el nivel de consumo óptimo, decimos que existen incentivos para que el niño estudie en el período 1; aumentando así las posibilidades de consumo del hogar en el periodo 2. Estas dos posibilidades se representan en los Gráficos 2 y 3 respectivamente.

Nótese que un aumento en la discriminación laboral, es decir un aumento de $\delta$ hace que el jefe del hogar de un hijo afrodescendiente tenga mayores incentivos para que su hijo trabaje en $t=1$ en vez de estudiar. Dado una dotación inicial en la sociedad, los hogares tomarán la decisión más racional. El punto A representa una elección cuando no existe discriminación en el mercado laboral. Es decir, los hogares envían los niños al sistema educativo para aumentar en consumo futuro $\left(C_{2}\right)$. El incremento de genera $\delta$ un cambio en la restricción presupuestaria, como se muestra en el Gráfico 4. Ahora, el punto B; en donde aumenta el consumo presente y disminuye el consumo futuro, resulta ser la elección óptima del jefe del hogar.

De lo anterior, se puede concluir, que bajo discriminación en el mercado laboral, crece el trabajo infantil. Es decir, la discriminación aumenta el costo de oportunidad $\delta$ de estudiar en el primer período.

\section{Conclusiones}

Encontramos una brecha en la probabilidad de trabajo infantil entre Afrocolombianos y No Afrocolombianos de 3,2\%, con una probabilidad más alta de 


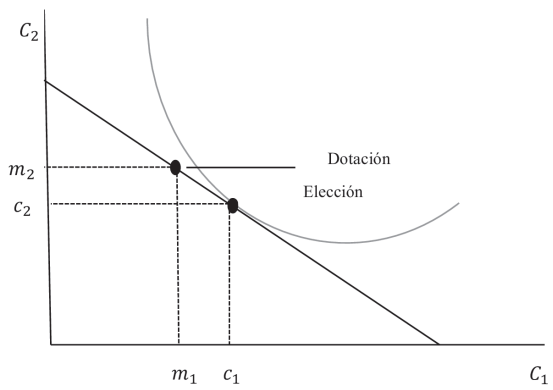

Figura 2: Posibilidad Consumo Uno 


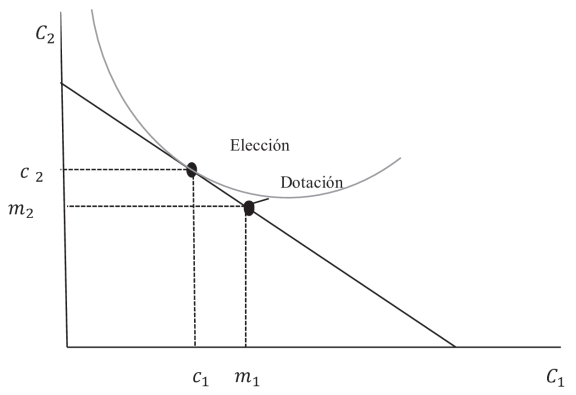

Figura 3: Posibilidad Consumo dos 


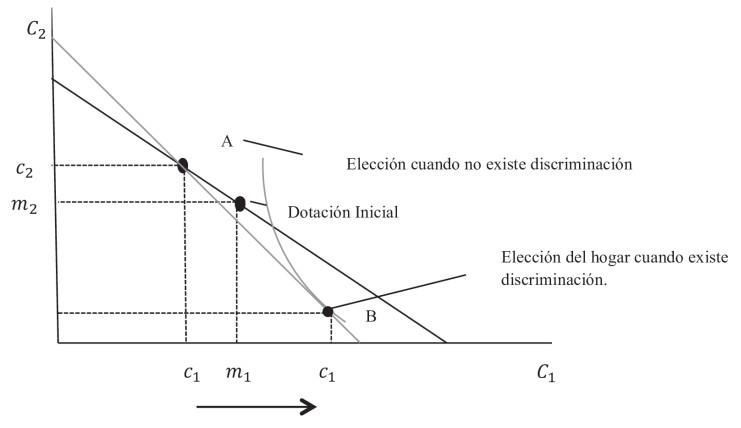

Figura 4: Estática Comparativa 
trabajo infantil para el primero de los grupos.

$\mathrm{Al}$ descomponer esta brecha en efecto características y efecto coeficientes se observa que el efecto características la define casi en su totalidad al explicar el 91,6\% de la misma. Lo anterior quiere decir que si le otorgáramos al grupo de los niños Afrocolombianos las características del grupo de niños no Afrocolombianos la diferencia de la tasa de trabajo infantil pasaría de 3,2\% a 0,29\%. Dentro de las características socioeconómicas utilizadas en el estudio, la ubicación geográfica del hogar, así como el nivel educativo del jefe de hogar resultan ser las principales características que explican la brecha. Lo hacen en $46 \%$ y $29,6 \%$ respectivamente.

En cuanto al efecto coeficientes, y en atención a la posible discriminación laboral en contra de la población Afrocolombiana, el modelo teórico presentado muestra cómo dicha posible discriminación podría ser una de las razones por las cuales hogares Afrocolombianos que logran anticipar dicho comportamiento prefieren que sus hijos se inserten rápidamente en el mercado laboral y no vayan a estudiar.

La posible presencia de discriminación que señala el trabajo, puede ser entendida desde el marco de la discriminación estadística, es decir, la existencia de asimetrías en la información en el mercado laboral. Esta discriminación puede generar, por un lado, procesos de autoselección de los afrocolombianos en trabajos de baja remuneración y, por otro lado, que los empleadores supongan que los afrocolombianos no presentan la competencia para ejercer cargos de alta remuneración; lo cual influye en la decisión de los jefes de hogares Afrocolombianos.

En términos de política económica, los resultados del trabajo sugieren la necesidad de políticas encaminadas a la mejora de las condiciones de calidad de vida en las zonas rurales. Mejoras en la calidad y en las expectativas de vida en las zonas rurales del país están asociadas con reducciones en la tasa de trabajo infantil.

Finalmente, es importante recordar que este estudio no pretende analizar la posible relación de causalidad entre raza y trabajo infantil. Aquí nos hemos limitado a una descomposición de la diferencia en tasas de trabajo infantil entre dos grupos, Afrocolombianos y no Afrocolombianos, para estudiar qué características socioeconómicas están más asociadas a dicha diferencia. Investigaciones futuras con énfasis en el efecto causal de raza sobre trabajo infantil requieren de más y mejor información. 


\section{Referências Bibliográficas}

Admassie, A. (2003), 'Child labour and schooling in the context of a subsistence rural economy: Can they be compatible?', International Journal of Educational Development 23(2), 167 - 185.

Arrow \& Kenneth, J. (1972), Models of job discrimination, in A. H. Pascal, ed., 'Racial Discrimination in Economic Life', Lexington Books, Massachusetts. D. C. Heath, pp. $83-102$.

Basu, K. \& Van, P. H. (1998), 'The economics of child labor', The American Economic Review 88(3), 412 - 427.

Becker, G. S. (1971), The Economics of Discrimination, 2 edn, The University of Chicago Press, Chicago and London.

Bernal, R. \& Cárdenas, M. (2006), Trabajo infantil en Colombia, Mercado laboral, pobreza y protección social, Fedesarrollo.

Blinder, A. S. (1973), 'Wage discrimination: Reduced form and structural estimates', The Journal of Human Resources 8(4), 436 - 455.

Emerson, P. M. \& Knabb, S. D. (2006), 'Opportuniy, inequality and the intergenerational transmission of child labour', Economica 73(291), $413-434$.

Ersado, L. (2002), Child labor and school decisions in urban and rural areas: Cross country evidence, Food Consumption and Nutrition Division Discussion Paper Brief 145, International Food Policy Research Institute, Washington, D.C.

Fairlie, R. (2005), 'An extension of the Oaxaca Blinder decomposition technique to logit and probit models', Journal of Economic and Social Measurement 30, $305-316$.

Fortin, N., Lemieux, T. \& Firpo, S. (2011), Decomposition methods in economics, Working Paper 16045, National Bureau of Economic Research.

Gradín, C. (2007), Why is poverty so high among afro-brazilians? a decomposition analysis of the racial poverty gap, IZA Discussion Papers 2809, Institute for the Study of Labor ( IZA ).

Gradín, C. (2010), Race and income distribution: Evidence from the US, Brazil and South Africa, Working Papers 179, ECINEQ, Society for the Study of Economic Inequality.

Kassouf, A. L., Mckee, M. \& Mossialos, E. (2001), 'Early entrance to the job market and its effects on adulth health: Evidence from Brazil, health policy and planning.', Health Policy and Planning 16(1), 21 -28.

Levy, V. (1985), 'Child labor and fertility behavior in a farming economy: Rural eypt', Economic Development and Cultural Change 33(4), 777 - 791.

Marx, C. (1999), El capital: Critica de la Economia Politica., 4 edn, Fondo de Cultura Economica.

Myers, W. (2001), Valuing diverses approaches to child labour, in B. White \& K. Lieten, eds, 'Child Labour Policy options', Aksant Academic Publishers. 
Ndjanyou, L. \& Djiénouassi, S. (2010), 'Characteristics and determinants of child labour in Cameroon', CSAE Conference: Economic Development in Africa

Oaxaca, R. (1973), 'Male-female wage differentials in urban labor markets', International Economic Review 14, 693 - 709.

O’Donnel, O., Rosati, F. \& Van Doorslaer, E. (2002), Child labor and health: Evidence and research issues, UCW Working Paper 1, Understanding Children's Work (UCW Programme).

Ofcina Internacional del Trabajo (2013), Medir los progresos en la lucha contra el trabajo infantil: Estimaciones y tendencias mundiales entre 2000 y 2012, ILO Publications Bureau, Ginebra.

Pedraza, A. \& Ribero, R. (2005), 'El trabajo infantil y juvenil en colombia y algunas de sus consecuencias claves', Coyuntura Social (33), 81 - 101.

Pedraza, A. \& Ribero, R. (2006), 'El trabajo infantil y juvenil en colombia $\mathrm{y}$ algunas de sus consecuencias claves', Revista Latinoamericana de Ciencias Sociales, Niñez y Juventud 4(1), 1 - 28.

Portilla, D. A. (2003), Mercado laboral y discriminación racial: una aproximación para cali, Documento CEDE 14, Universidad de los Andes, Bogotá.

Ray, R. (2000a), 'Analysis of child labour in Peru and Pakistan: a comparative study', Journal of Population Economics 13(1), 3 - 19.

Ray, R. (2000b), 'Poverty, household size and child welfare in india', Economic and Political Weekly 35(39), $3511-3520$.

Ray, R. (2001), 'Simultaneous analysis of child labour and child schooling: Comparative evidence from nepal and pakistan', Economic and Political Weekly 37(52), $5215-5224$.

Swaminathan, M. (1988), 'Economic growth and the persistence of child labor: Evidence from an indian city', World Development 26(8), 1513 - 1528.

Tenjo, J. \& Herrera, P. (2009), Los ensayos sobre discriminación: Discriminación salarial y discriminación en acceso al empleo por origen Étnico y por género, Colección Documentos de Economia 1, Universidad Javeriana, Bogotá.

Tharmmapornphilas, R. (2008), The Determinants of Child Labor in Thailand: The Role of Household, Public Policies and Exogenous Events, Disertación, Columbia University.

Tovar, L., Urueña, S. \& Castillo, M. (2009), 'Determinante del trabajo infantil y la escolaridad: el caso del valle del cauca en Colombia', Revista Latinoamericana de ciencias sociales, niñez y juventud 7(2), 707 - 733.

Viáfara, C. A. \& Urrea, F. (2006), 'Efectos de la raza y el género en el logro educativo y estatus socioocupacional para tres ciudades colombianas', Desarrollo y Sociedad $\mathbf{5 8 .}$ 
Wahba, J. (2000), Do Market Wages Influence Child Labor And Child Schooling? Department of Economics University of Southampton, Paper from the department of economics university of southampton, The World Bank.

Yun, M. (2003), 'Decomposing differences in the first moment', Economics Letters 82(2), $275-280$.

Yun, M. (2005), 'A simple solution to the identification problem in detailed wage decompositions', Economic Inquiry 4(766 -772).

Zapata, D., Contreras, D. \& Kruger, D. (2011), 'Child labor and schooling in Bolivia: Who's falling behind? the roles of domestic work, gender and ethnicity', World Development 39(4), 588 - 599.

Ávila, D. M. L. (2009), Pobreza y trabajo infantil: diferencias entre trabajo dentro y fuera del hogar. evidencia para Colombia, 2001-2003, Documentos de economia, Universidad Javeriana, Bogotá. 


\section{Apêndice A Anexos}

Tabela A.1: Tasa de Trabajo Infantil Según la Región de Origen

\begin{tabular}{lcc}
\hline Región & Trabaja (\%) & No Trabaja (\%) \\
\hline Atlántico & 8,9 & 91,1 \\
Oriental & 15,5 & 84,6 \\
Central & 15,6 & 84,5 \\
Pacífica & 21 & 79 \\
Bogotá & 7,3 & 92,7 \\
Antioquia & 14,6 & 85,4 \\
Valle del Cauca & 11,3 & 88,7 \\
San Andrés y Providencia & 4,8 & 95,2 \\
Orinoquía y Amazonía & 13 & 87 \\
\hline
\end{tabular}

Fuente: ECV 2003. Cálculos Propios.

Tabela A.2: Porcentaje de Hogares Pobres Según Región

\begin{tabular}{lcc}
\hline Región & Pobre (\%) & No Pobre (\%) \\
\hline Atlántico & 71,1 & 28,9 \\
Oriental & 64,9 & 35,1 \\
Central & 76,2 & 23,8 \\
Pacífica & 79,6 & 20,4 \\
Bogotá & 41,5 & 58,5 \\
Antioquia & 68,1 & 31,9 \\
Valle del Cauca & 53,8 & 46,2 \\
San Andrés y Providencia & 29,8 & 70,2 \\
Orinoquía y Amazonía & 54,6 & 45,4 \\
\hline Fuente: ECV 2003. Cálculos Propios.
\end{tabular}

Fuente: ECV 2003. Cálculos Propios. 


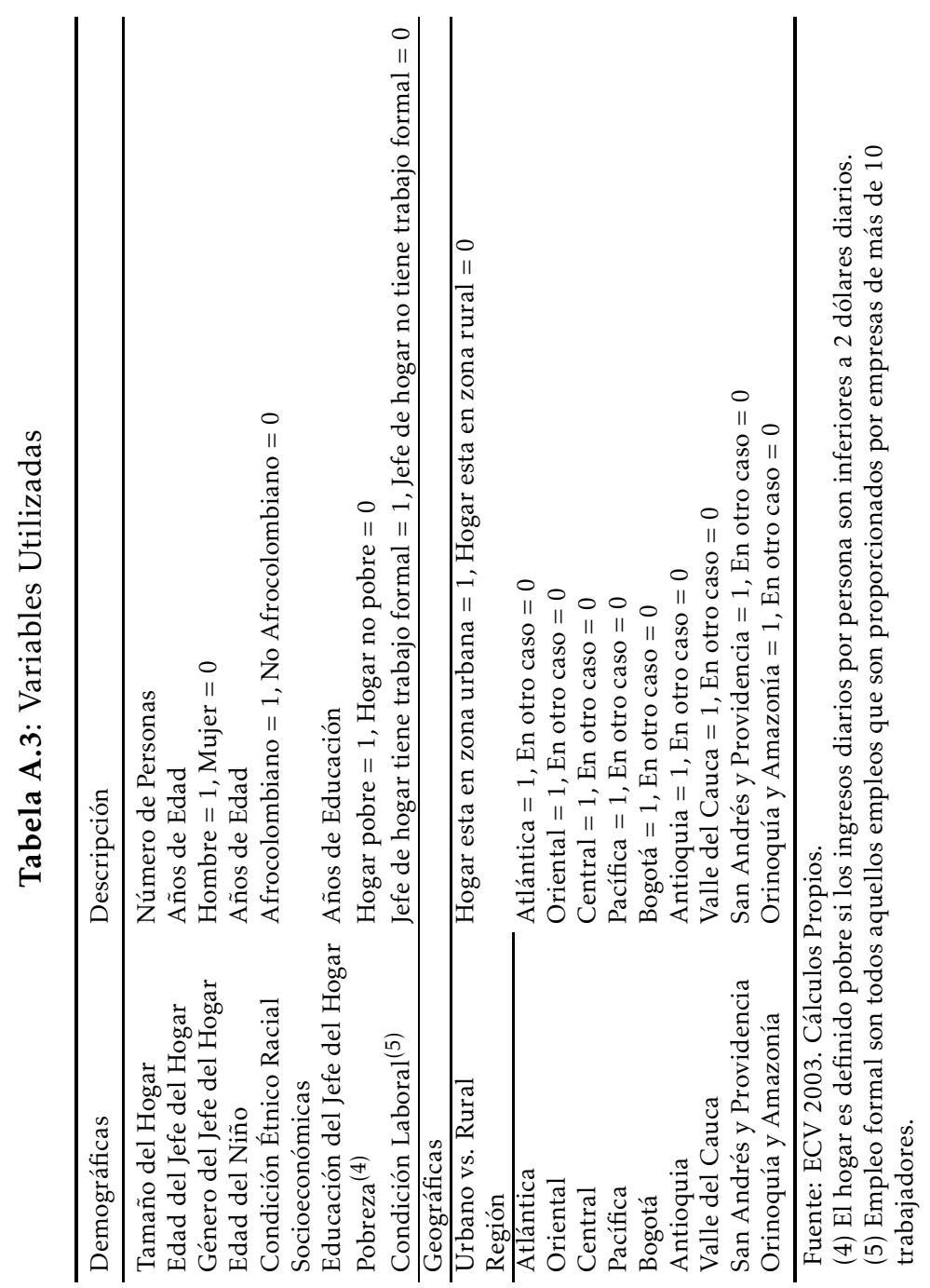


Tabela A.4: Tasa de trabajo Infantil y la Diferencia por Condición Étnico-Racial

\begin{tabular}{lc}
\hline Condición Étnico-Racial & Trabajo Infantil (\%) \\
\hline No Afrocolombiano & 11,8 \\
Afrocolombiano & 15,0 \\
Diferencia & 3,2 \\
\hline Fuente: ECV 2003. Cálculos Propios.
\end{tabular}

Tabela A.5: Raza: descomposición Efecto-características y Efecto-coeficientes

\begin{tabular}{|c|c|c|c|c|}
\hline & Coef. & $P>|z|$ & Coef. & $P>|z|$ \\
\hline Afrocolombiano & 0,149660 & 0,000 & 0,149660 & 0,000 \\
\hline No Afrocolombianos & 0,117546 & 0,000 & 0,117546 & 0,000 \\
\hline Diferencia & 0,032215 & 0,000 & 0,032215 & 0,000 \\
\hline Efecto-características & 0,029497 & 0,000 & 0,029497 & 0,000 \\
\hline Efecto-coeficientes & 0,002718 & 0,869 & 0,002718 & 0,869 \\
\hline & \multicolumn{2}{|c|}{ Efecto-características } & \multirow{2}{*}{\multicolumn{2}{|c|}{$\begin{array}{c}\text { Efecto-coeficientes } \\
8,4 \%\end{array}$}} \\
\hline Variables & $91,6 \%$ & & & \\
\hline Demográficas & $3,1 \%$ & \multicolumn{3}{|c|}{$-33,5 \%$} \\
\hline Tamaño del Hogar & $\begin{array}{c}0,00317 \\
9,8 \%\end{array}$ & 0,000 & $\begin{array}{c}-0,00003 \\
-0,1 \%\end{array}$ & 0,984 \\
\hline Edad del Jefe del Hogar & $\begin{array}{c}0,000708 \\
2,2 \%\end{array}$ & 0,348 & $\begin{array}{c}0,001348 \\
4,2 \%\end{array}$ & 0,732 \\
\hline Género del Jefe del Hogar & $\begin{array}{c}-0,000034 \\
-0,1 \%\end{array}$ & 0,857 & $\begin{array}{c}-0,000114 \\
-0,4 \%\end{array}$ & 0,741 \\
\hline Edad del niño & $\begin{array}{c}-0,002865 \\
-8,9 \%\end{array}$ & 0,203 & $\begin{array}{c}-0,011986 \\
-37,2 \%\end{array}$ & 0,681 \\
\hline Género del niño & $\begin{array}{c}0,000002 \\
0,0 \% \\
\end{array}$ & 0,931 & $\begin{array}{c}0,000000 \\
0,0 \% \\
\end{array}$ & 0,939 \\
\hline Educación, Actividad y Pobreza & $36,7 \%$ & $-9,8 \%$ & & \\
\hline Nivel educativo del Jefe & $\begin{array}{c}0,009534 \\
29,6 \%\end{array}$ & 0,000 & $\begin{array}{c}-0,002774 \\
-8,6 \%\end{array}$ & 0,683 \\
\hline Pobreza & $\begin{array}{c}0,000039 \\
0,1 \%\end{array}$ & 0,952 & $\begin{array}{c}-0,000317 \\
-1,0 \%\end{array}$ & 0,692 \\
\hline Condición Laboral & $\begin{array}{c}0,002245 \\
7,0 \% \\
\end{array}$ & 0,000 & $\begin{array}{c}-0,000065 \\
-0,2 \% \\
\end{array}$ & 0,917 \\
\hline Geográficas & $51,8 \%$ & $51,7 \%$ & & \\
\hline Urbano vs Rural & $\begin{array}{c}0,014822 \\
46,0 \%\end{array}$ & 0,000 & $\begin{array}{c}0,000167 \\
0,5 \%\end{array}$ & 0,693 \\
\hline Valle vs Resto de Regiones & $\begin{array}{c}0,001874 \\
5,8 \% \\
\end{array}$ & 0,038 & $\begin{array}{c}0,000565 \\
1,8 \% \\
\end{array}$ & 0,699 \\
\hline Constante & & & $\begin{array}{c}0,015920 \\
49,4 \% \\
\end{array}$ & 0,685 \\
\hline
\end{tabular}

Fuente: ECV 2003. Cálculos Propios. 
Tabela A.6: Afrocolombianos: descomposición Efecto-características y Efectocoeficientes

\begin{tabular}{|c|c|c|c|c|}
\hline & Coef. & $P>|z|$ & Coef. & $P>|z|$ \\
\hline Afrocolombiano & 0,1496599 & 0,000 & 0,1496599 & 0,000 \\
\hline No Afrocolombianos & 0,1175455 & 0,000 & 0,1175455 & 0,000 \\
\hline Diferencia & 0,0321144 & 0,000 & 0,0321144 & 0,000 \\
\hline Efecto-características & 0,0318682 & 0,000 & 0,0318682 & 0,000 \\
\hline Efecto-coeficientes & 0,0002462 & 0,976 & 0,0002462 & 0,976 \\
\hline \multirow[b]{2}{*}{ Variables } & \multicolumn{2}{|c|}{ Efecto-características } & \multicolumn{2}{|c|}{ Efecto-coeficientes } \\
\hline & \multicolumn{2}{|l|}{$99,2 \%$} & \multicolumn{2}{|l|}{$0,8 \%$} \\
\hline Demográficas & $2,5 \%$ & \multicolumn{3}{|c|}{$-3,2 \%$} \\
\hline Tamaño del Hogar & $\begin{array}{c}0,00334 \\
10,4 \%\end{array}$ & 0,000 & 0,00001 & 0,978 \\
\hline Edad del Jefe del Hogar & $\begin{array}{c}0,00067 \\
2,1 \%\end{array}$ & 0,351 & $\begin{array}{c}0,00015 \\
0,5 \%\end{array}$ & 0,976 \\
\hline Género del Jefe del Hogar & $-0,00006$ & 0,765 & $-0,00003$ & 0,976 \\
\hline Edad del niño & $\begin{array}{c}-0,00314 \\
-9,8 \%\end{array}$ & 0,186 & $\begin{array}{c}-0,00114 \\
-3,5 \%\end{array}$ & 0,976 \\
\hline Género del niño & $\begin{array}{c}0,00000 \\
0,0 \%\end{array}$ & 0,883 & $\begin{array}{c}-0,00002 \\
-0,1 \%\end{array}$ & 0,976 \\
\hline Educación, Actividad y Pobreza & $39,6 \%$ & \multicolumn{3}{|c|}{$0,6 \%$} \\
\hline Nivel educativo del Jefe & $\begin{array}{c}0,00974 \\
30,3 \%\end{array}$ & 0,000 & $\begin{array}{c}0,00031 \\
1,0 \%\end{array}$ & 0,976 \\
\hline Pobreza & $\begin{array}{c}0,00076 \\
2,4 \%\end{array}$ & 0,250 & $\begin{array}{c}-0,00012 \\
-0,4 \%\end{array}$ & 0,976 \\
\hline Condición Laboral & $\begin{array}{c}0,00222 \\
6,9 \%\end{array}$ & 0,000 & $\begin{array}{c}0,00001 \\
0,0 \% \\
\end{array}$ & 0,977 \\
\hline Geográficas & $57,1 \%$ & $3,4 \%$ & & \\
\hline Urbano vs Rural & $\begin{array}{c}0,01638 \\
51,0 \%\end{array}$ & 0,000 & $\begin{array}{c}0,00010 \\
0,3 \%\end{array}$ & 0,976 \\
\hline Valle vs Resto de Regiones & $\begin{array}{c}0,00196 \\
6,1 \%\end{array}$ & 0,052 & $\begin{array}{c}-0,00003 \\
-0,1 \%\end{array}$ & 0,976 \\
\hline Constante & & & $\begin{array}{c}0,00101 \\
3,1 \%\end{array}$ & 0,976 \\
\hline
\end{tabular}

Fuente: ECV 2003. Cálculos Propios. 
Tabela A.7: No Afrocolombianos: descomposición Efecto-características y Efecto-coeficientes

\begin{tabular}{|c|c|c|c|c|}
\hline & Coef. & $P>|z|$ & Coef. & $P>|z|$ \\
\hline Afrocolombiano & 0,14966 & 0,000 & 0,14966 & 0,000 \\
\hline No Afrocolombianos & 0,11755 & 0,000 & 0,11755 & 0,000 \\
\hline Diferencia & 0,03211 & 0,000 & 0,03211 & 0,000 \\
\hline Efecto-características & 0,02010 & 0,000 & 0,02010 & 0,000 \\
\hline Efecto-coeficientes & 0,01201 & 0,145 & 0,01201 & 0,145 \\
\hline & \multicolumn{2}{|c|}{ Efecto-características } & \multicolumn{2}{|c|}{ Efecto-coeficientes } \\
\hline Variables & $62,6 \%$ & \multicolumn{3}{|c|}{$37,4 \%$} \\
\hline Demográficas & $11,1 \%$ & \multicolumn{3}{|c|}{$-111,6 \%$} \\
\hline Tamaño del Hogar & $\begin{array}{c}0,00438 \\
13,6 \%\end{array}$ & 0,099 & $\begin{array}{c}0,00040 \\
1,2 \%\end{array}$ & 0,917 \\
\hline Edad del Jefe del Hogar & $\begin{array}{c}0,00065 \\
2,0 \%\end{array}$ & 0,367 & $\begin{array}{c}0,00499 \\
15,5 \%\end{array}$ & 0,463 \\
\hline Género del Jefe del Hogar & $\begin{array}{c}0,00024 \\
0,7 \%\end{array}$ & 0,699 & $\begin{array}{c}-0,00120 \\
-3,7 \%\end{array}$ & 0,639 \\
\hline Edad del niño & $\begin{array}{c}-0,00173 \\
-5,4 \%\end{array}$ & 0,196 & $\begin{array}{c}-0,03924 \\
-122,2 \%\end{array}$ & 0,059 \\
\hline Género del niño & $\begin{array}{c}0,00004 \\
0,1 \% \\
\end{array}$ & 0,751 & $\begin{array}{c}-0,00081 \\
-2,5 \% \\
\end{array}$ & 0,628 \\
\hline Educación, Actividad y Pobreza & $8,0 \%$ & \multicolumn{3}{|c|}{$29,3 \%$} \\
\hline Nivel educativo del Jefe & $\begin{array}{c}0,00264 \\
8,2 \%\end{array}$ & 0,295 & $\begin{array}{c}0,01242 \\
38,7 \%\end{array}$ & 0,108 \\
\hline Pobreza & $\begin{array}{c}-0,00241 \\
-7,5 \%\end{array}$ & 0,306 & $\begin{array}{c}-0,00333 \\
-10,4 \%\end{array}$ & 0,272 \\
\hline Condición Laboral & $\begin{array}{c}0,00235 \\
7,3 \% \\
\end{array}$ & 0,095 & $\begin{array}{c}0,00031 \\
1,0 \% \\
\end{array}$ & 0,798 \\
\hline Geográficas & $43,4 \%$ & \multicolumn{3}{|c|}{$119,8 \%$} \\
\hline Urbano vs Rural & $\begin{array}{c}0,01475 \\
45,9 \%\end{array}$ & 0,000 & $\begin{array}{c}0,00431 \\
13,4 \%\end{array}$ & 0,263 \\
\hline Valle vs Resto de Regiones & $\begin{array}{c}-0,00080 \\
-2,5 \% \\
\end{array}$ & 0,739 & $\begin{array}{c}-0,00036 \\
-1,1 \% \\
\end{array}$ & 0,352 \\
\hline Constante & & & $\begin{array}{c}0,03452 \\
107,5 \%\end{array}$ & 0,113 \\
\hline
\end{tabular}

Fuente: ECV 2003. Cálculos Propios. 